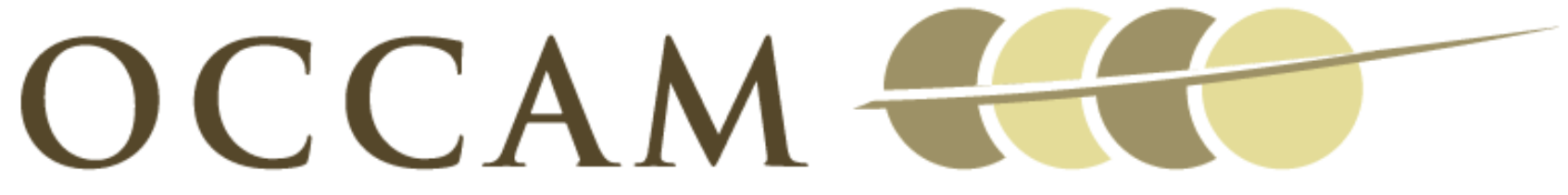

OXFORD CENTRE FOR COLLABORATIVE APPLIED MATHEMATICS

Report Number 12/03

The Fourier transform of tubular densities

by

Chris B. Prior and Alain Goriely

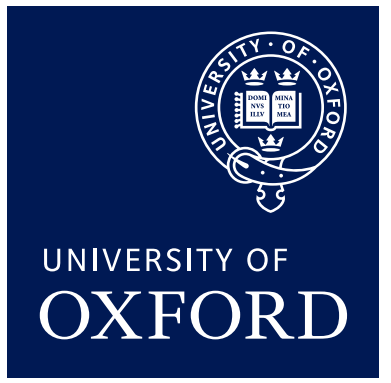

Oxford Centre for Collaborative Applied Mathematics Mathematical Institute

24 - 29 St Giles'

Oxford

OX1 3LB

England 



\title{
The Fourier transform of tubular densities
}

\author{
C B Prior ${ }^{1}$ and A Goriely ${ }^{2}$ \\ 12 Oxford centre for collaborative applied mathematics, Mathematical Institute 2429 St \\ Giles' Oxford OX1 3LB \\ E-mail: ${ }^{1}$ priorc@maths.ox.ac.uk \\ E-mail: ${ }^{2}$ goriely@maths.ox.ac.uk
}

\begin{abstract}
We consider the Fourier transform of tubular volume densities, with arbitrary axial geometry and (possibly) twisted internal structure. This density can be used to represent, among others, magnetic flux or the electron density of biopolymer molecules. We consider tubes of both finite radii and unrestricted radius. When there is overlap of the tube structure the net density is calculated using the super-position principle. The Fourier transform of this density is composed of two expressions, one for which the radius of the tube is less than the curvature of the axis and one for which the radius is greater (which must have density overlap). This expression can accommodate an asymmetric density distribution and a tube structure which has non-uniform twisting. In addition we give several simpler expressions for isotropic densities, densities of finite radius, densities which decay at a rate sufficient to minimise local overlap and finally individual surfaces of the tube manifold. These simplified cases can often be expressed as arclength integrals and can be evaluated using a system of first-order ODE'S.
\end{abstract}

PACS numbers: 02.30.Nw,02.40.Hw,02.40.Ma,87.15.bg,87.64.Bx,52.30.Cv

Submitted to: J. Phys. A: Math. Gen.

\section{Introduction}

Tubular densities are often used to represent physical systems including biopolymer molecules [38, 6, 5, 23], magnetic flux tubes (in the convective region of the sun $[36,39,33,30]$, in the coronal region $[41,42]$, in dynamo theory $[43,32,3]$; and mechanical and biological rods $[16,34,44,35,45])$. One way to probe or characterise the geometry of these objects is by using their spectral properties such as the Fourier transforms of their distributions.

In the case of bioploymers the density function would represent the electron density. A particularly important problem is the possibility of density overlap, which corresponds to the interaction of the atoms comprising the molecule. The radius of electron density is 
not required to be restricted, though it would drop to a negligible value at a significant radial distance from the axis. By comparison, for material rods, where the density would represent material points which cannot overlap through deformation, the tube would necessarily have a finite radius and a restriction on both the bending it can undergo and its overall shape so that it does not overlap. Recent theoretical work on biopolymers has linked these two physical scenarios. In particular various authors have provided evidence that one can characterise the geometry of known secondary protein structures, using a tubular electron density model for which the density has a finite radius and is forbidden to overlap (see $[38,5,6]$ and references therein). Briefly, the idea is that the geometry of the protein can be understood in terms of self-attraction, due to the need of hydrophilic sections of the molecule to be shielded from the surrounding water molecules, and the electromagnetic self-repulsion of the electron density. The self-repulsion is represented by the radius of the tube. It has been shown that this simple model predicts the formation of the ubiquitous alpha-helix structure, as a tube of finite radius which is helically coiled in a compact fashion [4] (see Figure 1). Another aspect of biopolymer structures, specifically proteins, is that a significant percentage of known tertiary structures are composed of helical geometries, specifically the alpha-helix and beta-sheet structures. According to Fitzkee et al, "approximately half the structure of folded proteins is either $\alpha$-helix or $\beta$-strands" [17]. Thus, one could characterise significant amounts of complex biopolymer structure in terms of a basic helical tubular units which have been rotated and translated in space. This idea was used by Hausrath and Goriely [22] to propose a continuous model for protein molecules which could be used search the space of possible tertiary protein geometries in an efficient fashion.

Mathematically, a tubular surface is defined by a spacecurve, representing the axis of the surface, and a set of circles centred on the axis and lying in its normal plane (see (a) and (b) in Figure 2). If we restrict the radius of the circles it is always possible to define the tube such that there is no overlap. That is to say each point on the tube could be described by a unique set of parameters, the arclength along the curve $s$, the radius of the circle $R$ and the angle $\theta$ made in the normal plane by a radial line joining the axis to a point on the circle. By varying the radius to the maximum value we can define a three dimensional manifold, constructed from a foliation of the tubes, on which there is a one-to-one relationship between the parameter space and the space in which the tube is embedded (see (c) in Figure 2). Of course if we do not restrict the radius of this manifold the parameter uniqueness of each point is (generally) lost due to overlap. In what follows are interested in tubular manifolds which may or may not overlap.

One can create a simple and versatile continuum model by defining a density on this tubular manifold as a continuous function $f(s, R, \theta)$, which assigns a value to each point $(s, R, \theta)$. The model would then be completed by providing rules which determine both the geometry of the tube structure and the value of the density. 
a

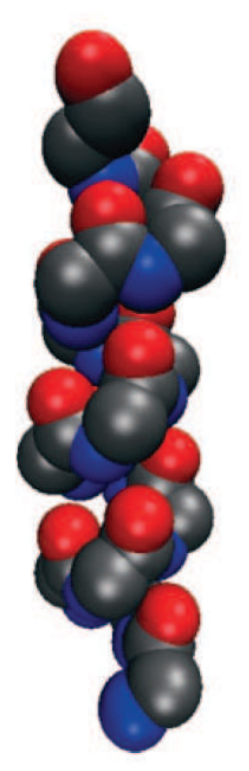

b

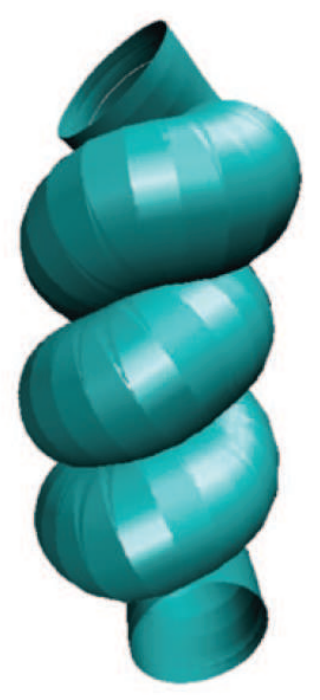

C

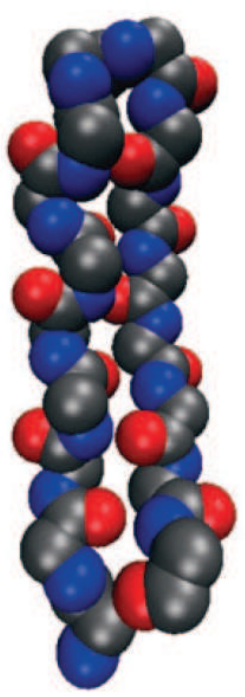

d

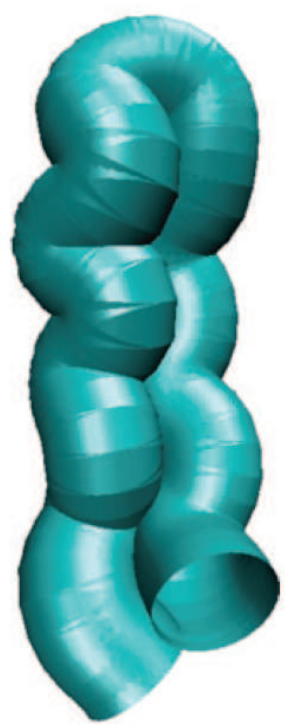

Figure 1. Figures (a) and (c) are depictions of an alpha helix (a) and two anti -parallel beta strand (c). These figures are a representation in which the molecules radius is represented by the van-der-walls radius of the constituent atoms. (b) and (c) are tube representations of the molecule. In [4] is is shown that the geometry of these structures can be captured by a continuous tube model (all figures are taken from [5]). One notes that the spherical molecules of figures (a) and (c) would fit inside the tube models (b) and (c).

(a)

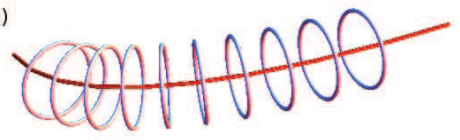

(b)

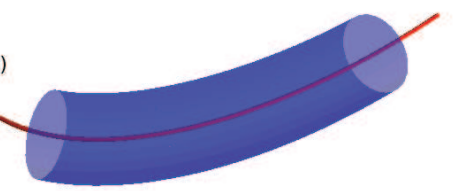

(c)

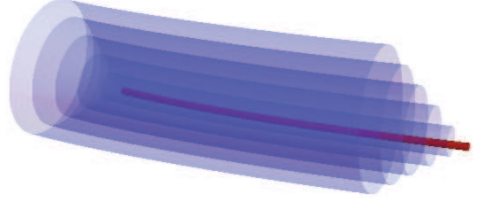

Figure 2. A depiction of the tubular manifold used in this note. In (a) we see a curve representing the tubular axis. It is surrounded by a set of circles which lie in the planes normal to the curve's tangent direction. If we consider a continuous set of such circles we obtain the tubular surface depicted in (b). We can create a manifold constructed form a set of tubular surfaces as shown in (c). A continuous set of such surfaces constitutes the type of tubular manifold, used in this note to define a tubular density. 
The structure of the tube itself can be important. In general magnetic flux tubes have an internal geometry, in that the field lines composing the tube tend to be twisted (a fact discussed in the references above). Theoretical work suggest that this inherent twisting is necessary for the tubes to remain stable in the sun's convective region [36, 29]. Moreover, a critical amount of twisting has been linked to instability of the flux tube structures in the coronal region $[41,42]$. Such inherent twisting can easily be incorporated into the tubular manifold description (see section 2).

The Fourier transform often plays an important role in various aspects of the theory surrounding the model. For biopolymer studies it is the mathematical tool underpinning the majority of structural identification techniques [15]. For this continuum model the relevant techniques would be medium/low resolutions methods such as small angle Xray scattering (SAXS) [40] and fiber diffraction [26] (see Hausrath and Goriely [23] for a discussion on the relevance of the continuous model in relation to imaging). In both cases the sample material is irradiated, and the resulting diffraction pattern can be interpreted in terms of the Fourier power spectrum of the model representing the biopolymer density.

In flux tube modelling the Fourier transform is used to characterise the energy and the flux-weighted geometry (helicity) of the flux tube $[31,30,3]$. The helicity of a flux tube can be expressed in terms of the amount of twisting about the tube axis of field lines comprising tube; and the global geometry of the tube's axis (the writhing) $[11,12,9,10]$. Particular theoretical importance is given to the scale of the helicity, where the scale can be interpreted in terms of the value of the Fourier wavevector $\mathbf{k}$. Small scales are associated with the twisting, the large scales correspond to the changes in global geometry (writhing) $[18,8,3]$. Possible models of the solar dynamo indicate that the development of dynamo fields in the sun is characterised by a conversion form small scale twisting to large scale writhing helicity $[43,32,8,3]$.

It is clear that the characterization of the global geometry of twisted and untwisted tube densities plays a central role in many models. One can, given a specific radius for the tube, define a restriction on the axial geometry of the tube which prevents local overlap due to extreme curvature of the tube (an example of such overlap is labelled local curvature on the tube (b) of Figure 3. However, tubes can still self-overlap even if this condition is satisfied (see the sections labelled non local overlap on (a) and (b) on Figure 3). A more restrictive condition on the axis geometry has been defined [20], which ensures there is no overlap. Both measures, intrinsic to the axis curve, underpin the tube biopolymer models discussed previously and can be incorporated as restrictions on our simple model. One can also relate the degree of twisting of the tube structure to its axial geometry. For tubes which close on themselves the sum of the writhing and twisting is a known invariant to deformations of the tube which prevent it from self-intersecting (see e.g. the proof of proposition 5 in [1]). So as the tube axis deforms the total twisting of the tube must also alter to conserve the total sum. This conservation law is an important consideration in 


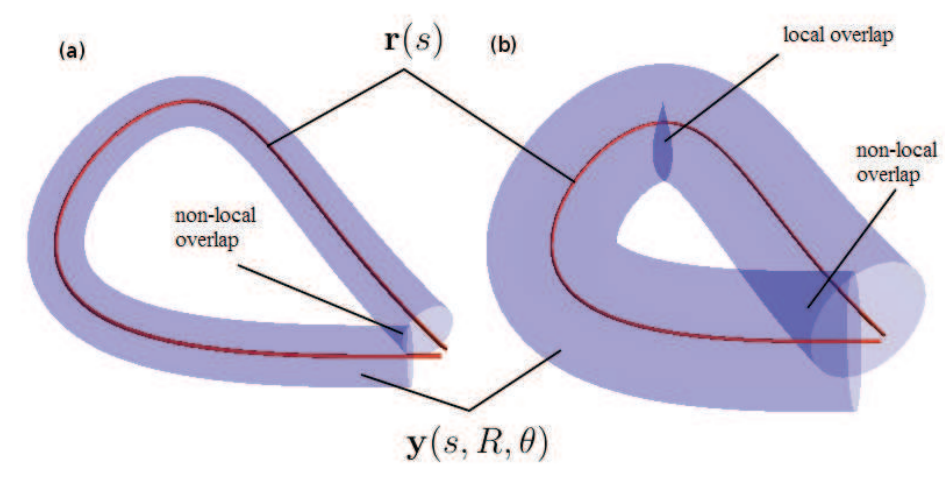

Figure 3. A depiction of the overlapping of tubular layers due to the non-zero curvature of the tube structure. The two figures have the same axis but differing radii. In (a) there is only one type of overlap which occurs due to points on the tube's axis being separated by less than the tube's radius, this is termed non-local overlap. In (b) this non local overlap has increased. In addition local overlap has occurred due to the extreme bending or curvature of the axis. Both types of overlap are determined by the axial geometry.

magnetic flux tube modelling $[9,19,37]$. Open analogues of this relationship also exist for open tube structures [10]. As shall be discussed, this conservation law could also naturally be applied to our density model.

The Fourier transform of tubular densities has not received much attention with regards to the geometric considerations discussed in the previous paragraph. AsgahriTarghi and Berger [3] considered the twist-writhe decomposition of the Fourier spectrum of a magnetic flux tube of uniform density. Their method for evaluating the transform is a numerical method which assumes that the tube can be defined in a box (a subset of $\mathbb{R}^{3}$ ) with periodic boundary conditions, which would require the tube's to be closed (no net flux in any specific direction over the volume of evaluation). The study was limited to the case in which the tubes were thin with no possibility of overlap. With regards to biopolymer literature most studies have concerned the transform of line densities, based on the celebrated transform expression for an infinite length helix and coiled coil [13, 14, 27], rather than the integration of volume densities. As such the issue of the effect of density overlap on the transform has not been considered.

There are several advantages to the model and transform expressions we propose in this note. First, we can consider arbitrary continuous densities and complex tubular geometry and define general transform expressions which cover all cases. In addition the expression can be subjected to an affine transformation in a simple manner; one might require this for complex biopolymer structures constructed form repeat helical units. Second, the model can be subjected to the type of topological and global geometrical restrictions discussed above. This allows the general transform expressions derived in this note to be applicable to a good range of physical applications. Third, the non-local density 
overlap is automatically calculated due to the fact that we define a density distribution which utilises the superposition principle (see Section 3).

The structure of this article is as follows. In Section 2 we introduce aspects of differential geometry of curves. We discuss the general form of the tubular structure which provides the frame for the molecular density. This includes a discussion on the choice of representing the tube manifold (the chosen frame) and how arbitrary twisting rates can be included. We discuss and define the concepts of local and non-local overlapping of the tube structure. Finally, we discuss the relationship between the tube Jacobian term and the concept of local overlapping.

In Section 3 we define a general density distribution (12). We first discuss the Fourier transform of tempered distributions, as our density definition falls into this particular class. The definition of the particular density distribution we employ concerns the rest of this section. This discussion incorporates the definition of a density function as a rapidly decreasing function (along the radial direction). We then introduce the full density, the combination of the density function and the tubular geometry which are convoluted using a Delta distribution. Finally we define the effect of applying an affine transformation to this density distribution (14).

In Section 4 we derive the most general expression for the Fourier transform of a tubular density. We split the derivation into two parts. First, the contribution to the transform from layers of the tubular density whose radius is such that there is no local overlap. The most general result is in this case is derived (26) we give explicit formulas when the density function is axially symmetric (27). We also derive an expression for a tube whose density is isotropic and constant in value. Second, we derive the contribution in the case in which there is local overlap of the tube. Finally we detail the general results for the Fourier transform of a single tubular surface, drawn from the full tubular manifold. These results are simple extensions of the more general full volume transforms.

In Section 5 we define conditions on the radial rate of decay of the density function in order that we can approximate the full transform accurately by only evaluating the contribution without local overlap. This greatly simplifies the required calculation.

\section{Definitions and Geometry}

\subsection{The frame}

We define the tube's axis as a non self-intersecting curve $\mathbf{r}(s):[0, L] \rightarrow \mathbb{R}^{3}, \mathbf{r} \in$ $\mathcal{C}^{3}\left([0, L] ; \mathbb{R}^{3}\right)$, where $s$ is the arclength. The tube's geometry is defined by a pair $\left(\mathbf{r}(s), \mathbf{d}_{1}(s)\right)$ where $\mathbf{d}_{1}(s):[0, L] \rightarrow \mathbb{R}^{3}, \mathbf{d}_{1} \in \mathcal{C}^{1}\left([0, L] ; \mathbb{R}^{3}\right)$, is a unit vector such that $\mathbf{r}^{\prime}(s) \cdot \mathbf{d}_{1}(s)=0$. A frame is a right-handed orthonormal basis of $\mathbb{R}^{3},\left(\mathbf{d}_{1}(s), \mathbf{d}_{2}(s), \mathbf{d}_{3}(s)\right)$, formed from the tangent vector $\mathbf{d}_{3}(s)=\mathbf{r}^{\prime}(s)$ and a third vector $\mathbf{d}_{2}(s)=\mathbf{d}_{3}(s) \times \mathbf{d}_{1}(s)$. The unit vectors $\mathbf{d}_{i}(s), i=1,2$, span the plane perpendicular to $\mathbf{d}_{3}$ as depicted in Figure 4. 


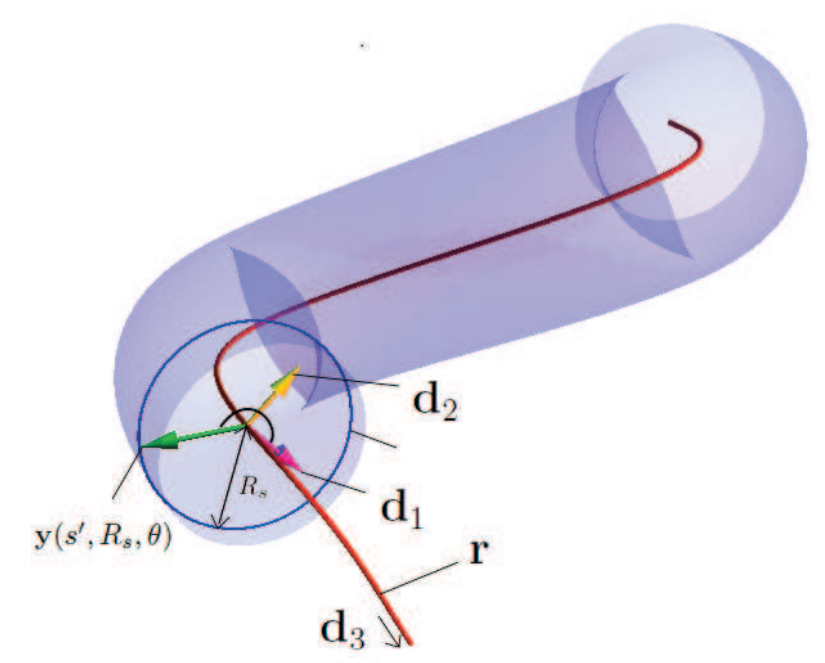

Figure 4. A depiction of the tube parametrisation. The backbone curve $\mathbf{r}$ is surrounded by a tube of radius $R$ (shown as an opaque surface). The moving orthonormal frame $\left(\mathbf{d}_{1}, \mathbf{d}_{2}, \mathbf{d}_{3}\right)$ is depicted. We see an example of a vector $\mathbf{y}\left(s^{\prime}, R_{s}, \theta\right)$ defined by a specific choice of $\theta$, given $s=s^{\prime}$ and $R=R_{s}$. The set $\theta \in S^{1}$ defines a set of vectors whose tip traces out the circle shown. The set of circles centred at $\mathbf{r}(s), s \in[0, L]$ is a tube surface of radius $R_{s}$ whose axial geometry is defined by $\mathbf{r}$.

\subsection{The tube}

We define a map $\mathbf{y}(s, R, \theta):[0, L] \times \mathbb{R}^{+} \times S^{1} \rightarrow \mathbb{R}^{3}$ as

$$
\mathbf{y}(s, R, \theta)=\mathbf{r}(s)+R\left(\mathbf{d}_{1}(s) \cos \theta+\mathbf{d}_{2}(s) \sin \theta\right) .
$$

The differentiability of this map follows from the differentiability of $\mathbf{r}$ and $\mathbf{d}_{1}$. Let $Y$ be the set of all such maps. For fixed values $s=s^{\prime}$ and $R=R_{s}$ the set of points defined by $y$ for all $\theta \in S^{1}$ define a circle of radius $R_{s}$, centred at $\mathbf{r}(s)$. The set of such circles for all $s \in[0, L]$ constitutes a tubular surface (see Figure 4) and the set of tubular surfaces for all $R_{s} \in\left[0, R_{m}\right]$ defines a tubular manifold. The density distribution is defined on this manifold. Since both $L$ and $R_{m}$ may be unbounded, this manifold is either a subset of $\mathbb{R}^{3}$ or the entire space. Within our framework, the triple $\left\{\mathbf{r}, \mathbf{d}_{1}, R_{m}\right\}$ defines a particular tubular manifold.

\subsection{Structure equations}

The evolution of the frame is determined by the rate of change in orientation of each vector $\mathbf{d}_{i}$ along $s$. This can be written as the vector product $\mathbf{d}_{i}^{\prime}(s)=\mathbf{u}(s) \times \mathbf{d}_{i}(s)$. The vector $\mathbf{u}$ is known as the Darboux vector and can be specified by three real valued functions $u_{j}(s), j=1,2,3$, where

$$
\mathbf{u}(s)=u_{1}(s) \mathbf{d}_{1}(s)+u_{2}(s) \mathbf{d}_{2}(s)+u_{3}(s) \mathbf{d}_{3}(s) .
$$


Here $u_{1}$ and $u_{2}$ represent curvatures of the the frame, so that $\sqrt{u_{1}^{2}+u_{2}^{2}}=\kappa$, the principal curvature. The function $u_{3}$ determines the rate at which the pair $\left(\mathbf{d}_{1}, \mathbf{d}_{2}\right)$ rotates about $\mathbf{d}_{3}$ (termed the twisting rate). We have the following system of ordinary differential equations

$$
\begin{aligned}
& \mathbf{d}_{1}^{\prime}=-u_{2} \mathbf{d}_{3}+u_{3} \mathbf{d}_{2}, \\
& \mathbf{d}_{2}^{\prime}=u_{1} \mathbf{d}_{3}-u_{3} \mathbf{d}_{1}, \\
& \mathbf{d}_{3}^{\prime}=-u_{1} \mathbf{d}_{2}+u_{2} \mathbf{d}_{1}, \\
& \mathbf{r}^{\prime}=\mathbf{d}_{3} .
\end{aligned}
$$

For a given Darboux vector $\mathbf{u}$, length $L$ and radius $R_{m}$, a tube manifold is defined by the solution of (3), up to a global translation and rotation determined by the initial conditions.

\subsection{Choice of frame}

Different choices of the vector $\mathbf{d}_{1}$ correspond to different frames. For instance the Frenet frame is obtained by the choice $\mathbf{d}_{1}=\mathbf{d}_{3}^{\prime} /\left|\mathbf{d}_{3}^{\prime}\right|$, the normal vector, and $\mathbf{d}_{2}=\mathbf{d}_{3} \times \mathbf{d}_{1}$ is the binormal vector. Equations (3) reduce then to the well known Frenet-Serret equation,

$$
\begin{aligned}
& \mathbf{d}_{3}^{\prime}=\kappa \mathbf{d}_{1}, \\
& \mathbf{d}_{1}^{\prime}=-\kappa \mathbf{d}_{3}+\tau \mathbf{d}_{2}, \\
& \mathbf{d}_{2}^{\prime}=-\tau \mathbf{d}_{1}, \\
& \mathbf{r}^{\prime}=\mathbf{d}_{3} .
\end{aligned}
$$

The torsion $\tau$ is defined as $\tau(s)=\mathbf{d}_{3}(s) \cdot\left(\mathbf{d}_{1}(s) \times \mathbf{d}_{1}^{\prime}(s)\right)$ and determined entirely by the tube's axial geometry. Hausrath and Goriely [22] have shown that the Frenet frame is a convenient choice of basis for searching the fold space of possible biopolymer backbone geometries in the case in which we assume they are constructed from helical segments, as the curvature and torsion of the helix are constant, leading to explicit solutions of the Frenet-Serret equation (4). The Frenet frame has drawback of being ill-defined if the derivative of the tangent vector vanishes.

Another consideration is the possibility of rotation of the frame which is independent of the axis geometry. This is important for magnetic flux tubes and twisted elastic rods in which the bulk density surrounding the axis can rotate independently of the axial deformation. The twist can be described by rotating the Frenet basis, i.e.

$$
\begin{aligned}
& \mathbf{d}_{1}=\cos (\omega(s)) \widehat{\mathbf{N}}+\sin (\omega(s)) \widehat{\mathbf{B}}, \\
& \mathbf{d}_{2}=-\sin (\omega(s)) \widehat{\mathbf{N}}+\cos (\omega(s)) \widehat{\mathbf{B}} .
\end{aligned}
$$

Here the differentiable function $\omega$ controls the additional rotation of the frame on top of the rotation of the Frenet basis due to torsion. If we identify the rotated frame (5) with the director basis then we have the following expressions for $u_{i}$

$$
u_{1}(s)=\kappa \sin (\omega), \quad u_{2}(s)=\kappa \cos (\omega), \quad u_{3}(s)=\tau+\omega^{\prime}(s) .
$$




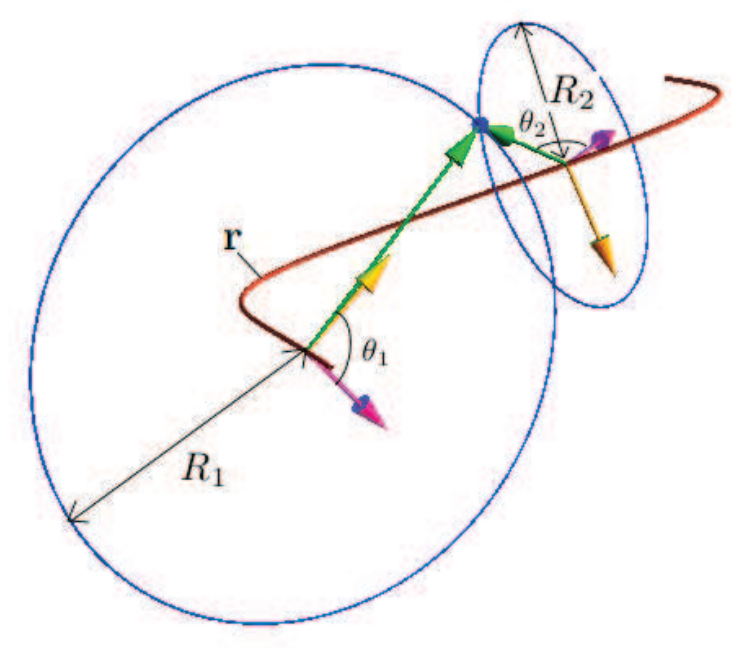

Figure 5. A depiction the overlap of tubes from differing parameter values of the tube structure $\mathbf{y}(s, R, \theta)$. The axis curve is shown in red. We consider a point in the ambient space marked as a blue dot. The two axis curve points corresponding to parameter sets $\left(s_{1}, R_{1}, \theta_{1}\right)$ and $\left(s_{2}, R_{2}, \theta_{2}\right)$ for which (12) is non-zero at this point are marked. The circular cross-section of the tube corresponding to these sets are shown to overlap at the point. Also displayed are the Frenet frame pair $\left(\mathbf{d}_{1}, \mathbf{d}_{2}\right)$ at $\mathbf{r}\left(s_{1}\right)$ and $\mathbf{r}\left(s_{2}\right)$.

This additional rotation could also be applied to other frames, such as the natural frame of Bishop [7] or the directional basis used to map the Euler angle rotations to the configuration of a material tube (rod) (see Antman [2] pg 315).

One might also consider the application of the twist-writhe topology to such structures. Berger and Prior [10] have shown that one can apply a directional frame which allows for the definition of directional analogues of the writhing and twisting measures discussed in the introduction.

In this note we define all expressions using an general basis $\left(\mathbf{d}_{1}, \mathbf{d}_{2}, \mathbf{d}_{3}\right)$. The effect of the choice of frame is shown to be limited to defining the functions $\left(u_{1}, u_{2}, u_{3}\right)$ and the evaluation of a function which involves the dot product of the Fourier vector $\mathbf{k}$ and the pair $\left(\mathbf{d}_{1}, \mathbf{d}_{2}\right)$ (Section 4.1).

\subsection{Tube overlap}

Readers familiar with the differential geometry of tubular structures will be aware that, unless $R$ has a restricted range, or the axis curve $\mathbf{r}$ is a straight line, there is no overlap of the tubes [24]. That is to say the tube map (1) is not generally injective and there are at least two distinct parameter sets, $\left(s_{i}, R_{i}, \theta_{i}\right)$ and $\left(s_{j}, R_{j}, \theta_{j}\right)$, for which $\mathbf{y}\left(s_{i}, R_{i}, \theta_{i}\right)=\mathbf{y}\left(s_{j}, R_{j}, \theta_{j}\right)$ (see Figure 5 ). There are two ways in which this overlap can 

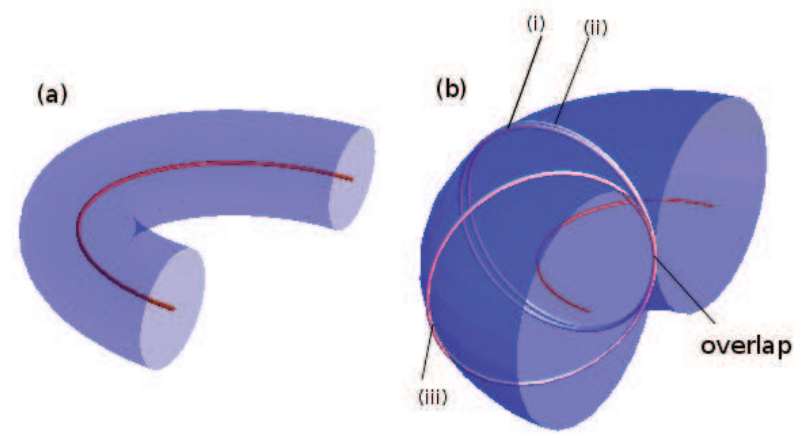

Figure 6. A depiction of the dependency of local overlap on the tube's radius and curvature. The red curves represent an axis curve $\mathbf{r}(s)=(\sin (\pi s), \cos (\pi s), s)$, for the range $s \in[0,1]$. The curvature is $\kappa(s)=1, \forall s \in[0,1]$. In (a) the tube's radius is $R=0.3$, there is no overlap. In (b) the radius is 1 , thus matching the critical curvature condition. We see the tube has an overlap point labelled on the figure. Because of the constant curvature this point is a site of local as well as non-local overlap. The circles labelled (i) and (ii) overlap and are in an $\epsilon$-neighbourhood of each other (there separation is exaggerated so as to be visible), this type of overlap is called local overlap in our discussion. Circle (iii) also overlaps circles (i) and (ii), however, as it is not in their $s$ neighbourhood this overlap classed as non-local, based on the definition in the text.

occur, locally and non-locally.

The local overlap can be defined from the curvature $\kappa$ at a point $\mathbf{r}\left(s_{i}\right)$. The inverse of $\kappa\left(s_{i}\right)$ can be interpreted geometrically as the radius $R_{i}$ of a sphere of which a single surface point touches the curve at $\mathbf{r}\left(s_{i}\right)$. This radius represents a critical radius for a tube surrounding $\mathbf{r}$ beyond which there is overlap [28]. For $R>R_{i}$ the circles in an $\epsilon$-neighbourhood of $s_{i}$ overlap (see Figure 6 ). We refer to overlap where $\epsilon$ is vanishingly small as local.

All other overlaps are referred to as non-local (see Figure 3 and Figure 6). Such overlap is a result of the global geometry of the curve and does not necessarily require that the curvature is particularly high at any point, though it must be non-zero for some $s$ domain. Gonzalez and Maddocks [20] extended this concept of the critical radius of curvature, to define the global radius of curvature, a critical radius for the tube structure below which no overlap occurs. We do not discuss this global condition in detail here as it does not explicitly affect the form of our general expressions. 


\subsection{The tube Jacobian}

The definition of the tubular density requires that we include the Jacobian of the tube. We shall consider both surface and volume Jacobian terms. We have

$$
J=\left|\frac{\partial \mathbf{y}(s, R, \theta)}{\partial(s, R, \theta)}\right|=R\left(1-R\left(u_{2} \cos \theta-u_{1} \sin \theta\right)\right) .
$$

The surface element Jacobian is identical with a fixed $R$ value. Alternatively we can write the two curvatures in polar coordinates as

$$
\begin{aligned}
& u_{1}(s)=\kappa(s) \sin \phi(s), \\
& u_{2}(s)=\kappa(s) \cos \phi(s) .
\end{aligned}
$$

Where $\phi(s)$ is real valued and differentiable. Using this convention we have

$$
J=\left|\frac{\partial \mathbf{y}(s, R, \theta)}{\partial(s, R, \theta)}\right|=R(1-R \kappa \cos (\theta+\phi)) .
$$

The specific form $\phi$ takes depends upon the construction of the frame. For example for the rotated Frenet frame defined in (5) we have $\phi=\omega$.

In our definition of density we require that the Jacobian is taken in absolute value to ensure positivity of the density when the manifold overlaps. For $R>1 / \kappa$, the function $|J|$ is non-differentiable with respect to $\theta$. This corresponds to the existence of local-overlap. Non-local overlap can occur for $R<1 / \kappa$ [20]. It is only the presence of local overlap that requires special handling when integration over $\theta$ is performed.

\section{Density and Fourier transform definitions}

\subsection{The Fourier transform of Distributions}

Since we consider the Fourier transform of densities we are interested in the Schwartz space of rapidly decreasing functions $\mathcal{S}\left(\mathbb{R}^{n}\right)=\left\{f \in \mathcal{C}^{\infty}\left(\mathbb{R}^{n}\right) \mid\|f\|_{\alpha, \beta}<\infty, \forall \alpha, \beta\right\}$, where,

$$
\|f\|_{\alpha, \beta}=\sup _{\mathbf{x} \in \mathbb{R}^{n}}\left|\mathbf{x}^{\alpha} D^{\beta} f(\mathbf{x})\right| .
$$

Here $\alpha$ and $\beta$ employ the multi-index notation and $D$ is the partial differential operator [25]. Tempered distributions constitute the space of all continuous linear maps $\mathcal{S}^{\prime}\left(\mathbb{R}^{n}\right)=$ $\left\{\mu: \mathcal{S}\left(\mathbb{R}^{n}\right) \rightarrow \mathbb{C}\right\}$.

We denote points in the ambient space $\mathcal{A}$ as $\mathbf{x} \in \mathbb{R}^{3}$. Points in the Fourier space $\mathcal{K}$ are denoted $\mathbf{k} \in \mathbb{R}^{3}$. In what follows we shall be interested in the Fourier transform, a continuous isomorphic map $F: \mathcal{S}^{\prime}\left(\mathbb{R}^{3}\right) \rightarrow \mathcal{S}^{\prime}\left(\mathbb{R}^{3}\right)$ defined by

$$
F(\mathbf{k})=\frac{1}{\sqrt{2 \pi}} \int_{\mathcal{A}} \mathrm{d} \mathbf{x} \mu(\mathbf{x}) \mathrm{e}^{\mathrm{i} \mathbf{k} \cdot \mathbf{x}}
$$

The restriction to tempered distributions means that $F$ is invertible,

$$
\mu(\mathbf{x})=\frac{1}{\sqrt{2 \pi}} \int_{\mathcal{K}} \mathrm{d} \mathbf{k} F(\mathbf{k}) \mathrm{e}^{-\mathrm{i} \mathbf{k} \cdot \mathbf{x}},
$$


and satisfies Parseval's theorem

$$
\int_{\mathcal{A}}|\mu(\mathbf{x})|^{2} \mathrm{~d} \mathbf{x}=\int_{\mathcal{K}}|F(\mathbf{k})|^{2} \mathrm{~d} \mathbf{k} .
$$

See e.g. [25].

\subsection{The tubular density distribution}

We define our volume densities using a convolution of a scalar density function and a delta-distribution. Let $\rho(s, R, \theta) \in \mathcal{S}\left(\mathbb{R}^{3}\right)$ be the density at a point on the tube manifold. To define the physical density at a point $\mathbf{x} \in \mathcal{A}$ we need to consider all values $\left\{\left(s_{i}, R_{i}, \theta_{i}\right)\right\}$ for which $\mathbf{y}\left(s_{i}, R_{i}, \theta_{i}\right)=\mathbf{x}$. Then the density is the super-position of all such parameter values. That is the density at a point $\mathbf{x} \in \mathcal{A}$ is generated by a function $\rho$ on a tube manifold $\left\{\mathbf{r}, \mathbf{d}_{1}, R_{m}\right\}$ via a continuous mapping $\mu: \mathcal{S}\left(\mathbb{R}^{3}\right) \times Y \times \mathbb{R}^{+} \rightarrow \mathbb{R}$, where

$\mu\left[\rho, \mathbf{y}, R_{m}\right](\mathbf{x})$

$=\int_{0}^{L} \mathrm{~d} s \int_{0}^{R_{\mathrm{m}}} \mathrm{d} R R \int_{0}^{2 \pi} \mathrm{d} \theta|1-R \kappa \cos (\theta+\phi)| \rho(s, R, \theta) \delta(\mathbf{x}-\mathbf{y}(s, R, \theta))$.

This mapping is a tempered distribution since it is a bounded linear map of a Schwartz function. The absolute operation on the Jacobian is necessary to ensure the density preserves the sign of $\rho(s, R, \theta)$ for all overlapped sections. We give a simple illustrative example of how this density is defined. Consider a radially unbound tube $\mathbf{y}_{c}(s, R, \theta)=$ $(R \cos (\theta), R \sin (\theta), s)$, which is defined with a rectilinear coordinate system and $\mathbf{r}(s)=$ $(0,0, s)$, thus $J=R$. We choose a density $\rho_{\exp }(s, R, \theta)=\alpha e^{-\beta R^{2}}$, where $\alpha$ and $\beta$ are constants. For a point in the ambient space $\mathbf{x}=(1,0,1)$ there is a single contribution from $\rho$ at $(s, R, \theta)=(1,1,0)$, giving $\mu(\mathbf{x})=\alpha e^{(-1 / \beta)}$. In this particular case there is one single parameter set $(s, R, \theta)$ for each point in $\mathcal{A}$, and applying $\mu$ to all $\mathbf{x} \in \mathcal{A}$ defines a cylindrically symmetric Gaussian density profile centred on the line $(0,0, s)$.

We can also consider the density restricted to a single tube surface of radius $R_{s}$. In this case we define a mapping $\mu_{s}: \mathcal{S}\left(\mathbb{R}^{3}\right) \times Y \times \mathbb{R}^{+} \rightarrow \mathbb{R}$ by

$\mu_{s}\left[\rho, \mathbf{y}, R_{s}\right](\mathbf{x})$

$=R_{s} \int_{0}^{L} \mathrm{~d} s \int_{0}^{2 \pi} \mathrm{d} \theta\left|1-R_{s} \kappa \cos (\theta+\phi)\right| \rho\left(s, R_{s}, \theta\right) \delta\left(\mathbf{x}-\mathbf{y}\left(s, R_{s}, \theta\right)\right)$.

Once again this mapping employs the super-position principle where there is overlap.

\subsection{Affine transformations}

If the density is known for a given tube manifold $\left\{\mathbf{r}, \mathbf{d}_{1}, R_{m}\right\}$ and function $\rho$, it is of interest to know the corresponding density after a rigid body motion of $\mathbf{y}$. To do this we define a constant linear transformation $\mathcal{R} \in G L(3, \mathbb{R})$ and a constant translation $\mathbf{x}_{\mathrm{c}} \in \mathbb{R}^{3}$. 
The affine map of a vector $\mathbf{x} \in \mathbb{R}^{3}$ is then specified by $\mathbf{x} \rightarrow \mathcal{R} \mathbf{x}+\mathbf{x}_{\mathrm{c}}$ and the volume density $\mu$ can then be written as

$\mu\left[\rho, \mathcal{R} \mathbf{y}+\mathbf{x}_{\mathrm{c}}, R_{m}\right](\mathbf{x})=$

$=\int_{0}^{L} \mathrm{~d} s \int_{0}^{R_{\mathrm{m}}} \mathrm{d} R R \int_{0}^{2 \pi} \mathrm{d} \theta|1-R \kappa \cos (\theta+\phi)| \rho \delta\left(\mathbf{x}-\mathcal{R} \mathbf{y}-\mathbf{x}_{\mathrm{c}}\right)$.

In section 4 we shall show that the general expression for $F[\mu]$ can incorporate such a transform in a particularly simple fashion. The same definition can be applied to the surface transform (13).

\section{Fourier transform of $\mu$}

Now that our density is defined we derive expressions for its Fourier transform. We consider a tubular density subject to an affine transformation. Inserting (14) into (11) we have

$$
\begin{gathered}
F\left[\rho, \mathcal{R} \mathbf{y}+\mathbf{x}_{\mathrm{c}}, R_{m}\right](\mathbf{k})=\frac{1}{\sqrt{2 \pi}} \int_{\mathcal{A}} \mathrm{d} \mathbf{x} \int_{0}^{L} \mathrm{~d} s \int_{0}^{R_{m}} \mathrm{~d} R \int_{0}^{2 \pi} \mathrm{d} \theta|J| \rho \delta\left(\mathbf{x}-\mathcal{R} \mathbf{y}-\mathbf{x}_{\mathbf{c}}\right) \mathrm{e}^{\mathrm{i} \mathbf{k} \cdot \mathbf{x}}, \\
=\frac{e^{\mathrm{i} \mathbf{k} \cdot \mathbf{x}_{\mathrm{c}}}}{\sqrt{2 \pi}} \int_{0}^{L} \mathrm{~d} s \int_{0}^{\infty} \mathrm{d} R \int_{0}^{2 \pi} \mathrm{d} \theta|J| \rho \mathrm{e}^{\{\mathbf{i} \mathbf{k} \cdot \mathcal{R} \mathbf{y}\}} .
\end{gathered}
$$

This is equivalent to a change of the basis of Fourier space. As discussed in Section 2.6 we must evaluate the $R$ integral on two distinct domains, $R<\kappa$ and $R \geq \kappa$ (assuming $\left.R_{m}>\kappa\right)$. On the domain $R \geq \kappa$ there are two points of $\theta \in S^{1}$ for which $1-\kappa R \cos (\theta+\phi)$ changes sign. This requires that we split the $\theta$ part of the integration on this $R$ domain. As $\kappa$ will in general have dependence on $s$, the length of the three $\theta$ domains will vary with $s$.

The general single integral expression we derive can be written as a sum of two functions. One representing the contribution from the subset of the tubular manifold for which there is no local overlap $(R<1 / \kappa)$ and one for its complement $(R \geq 1 / \kappa)$.

\subsection{No local overlap $R<1 / \kappa$}

We first consider $F_{\text {no }}$ (no overlap), the contribution to (15) with $R<\kappa$,

$$
F_{\text {no }}\left[\rho, \mathcal{R} \mathbf{y}+\mathbf{x}_{\mathrm{c}}, R_{m}\right](\mathbf{k})
$$

$=\frac{e^{\mathrm{i} \mathbf{k} \cdot \mathbf{x}_{\mathrm{c}}}}{\sqrt{2 \pi}} \int_{0}^{L} \mathrm{~d} s e^{\mathrm{i} \mathbf{k} \cdot \mathcal{R} \mathbf{r}} \int_{0}^{1 / \kappa} \mathrm{d} R R \int_{0}^{2 \pi} \mathrm{d} \theta(1-\kappa R \cos (\theta+\phi)) \rho \mathrm{e}^{\left\{\mathrm{i} R \mathbf{k} \cdot \mathcal{R}\left(\mathbf{d}_{1} \cos \theta+\mathbf{d}_{2} \sin \theta\right)\right\}}$,

where we have used (1). Since $\rho$ is periodic in $\theta$, we have

$$
\rho=\sum_{n=0}^{\infty} a_{n}(s, R) \cos (n \theta)+b_{n}(s, R) \sin (n \theta),
$$


where $a_{n}(s, R), b_{n}(s, R) \in \mathcal{S}\left(\mathbb{R}^{2}\right)$. We now consider the $\theta$ part of the integral. To simplify we make the change of variables $\theta=\psi+\delta$ and apply the following substitutions

$$
C_{1}=\mathbf{k} \cdot \mathcal{R} \mathbf{d}_{1}, \quad C_{2}=\mathbf{k} \cdot \mathcal{R} \mathbf{d}_{2}, \quad \tan \delta=-\frac{C_{1}}{C_{2}}, \quad C=\sqrt{C_{1}^{2}+C_{2}^{2}} .
$$

After applying these substitutions and (17) to (16) we must evaluate an infinite sum over $n$ of the following integrands

$$
\begin{aligned}
& \int_{\delta}^{2 \pi+\delta} \cos (n \psi+n \delta)(1-\kappa R \cos (\psi+\phi+\delta)) \mathrm{e}^{\mathrm{i} R C \sin \psi} \mathrm{d} \psi \\
& \int_{\delta}^{2 \pi+\delta} \sin (n \psi+n \delta)(1-\kappa R \cos (\psi+\phi+\delta)) \mathrm{e}^{\mathrm{i} R C \sin \psi} \mathrm{d} \psi
\end{aligned}
$$

We emphasise that $\phi$ only has a dependence on $s$ and is treated as constant for what follows. If we choose $\delta=\pi-\phi$ and re-label $\psi \rightarrow \theta$ the total $\theta$ integral can be constructed from the following two integrals

$$
\begin{aligned}
& (-1)^{n} a_{n} \int_{\pi-\phi}^{3 \pi-\phi}(\cos (n \theta) \cos (n \phi)+\sin (n \theta) \sin (n \phi))(1+\kappa R \cos \theta) \mathrm{e}^{\mathrm{i} R C(s) \sin \theta} \mathrm{d} \theta \\
& (-1)^{n} b_{n} \int_{\pi-\phi}^{3 \pi-\phi}(\sin (n \theta) \cos (n \phi)-\cos (n \theta) \sin (n \phi))(1+\kappa R \cos \theta) \mathrm{e}^{\mathrm{i} R C(s) \sin \theta} \mathrm{d} \theta .
\end{aligned}
$$

We show in Appendix A that (19) and (20) can be written in terms of Bessel functions, and (16) written as

$$
\begin{aligned}
& F_{\mathrm{no}}\left[\rho, \mathcal{R} \mathbf{y}+\mathbf{x}_{\mathrm{c}}, R_{m}\right](\mathbf{k})= \\
& \frac{\mathrm{e}^{\mathrm{i} \mathbf{k} \cdot \mathbf{x}_{\mathrm{c}}}}{\sqrt{2 \pi}} \sum_{n=0}^{\infty}(-1)^{n} \int_{0}^{L} \mathrm{~d} s \mathrm{e}^{\mathrm{i} \mathbf{k} \cdot \mathcal{R} \mathbf{r}} \int_{0}^{1 / \kappa} \mathrm{d} R R 2 \pi J_{n}(R C) \\
& \left\{\begin{array}{cc}
a_{n}\left(\cos (n \phi)+\frac{\mathrm{i} \kappa n}{C} \sin (n \phi)\right)+b_{n}\left(-\sin (n \phi)+\frac{\mathrm{i} \kappa n}{C} \cos (n \phi)\right) & \text { If } n \text { is even } \\
a_{n}\left(\mathrm{i} \sin (n \phi)+\frac{\kappa n}{C} \cos (n \phi)\right)+b_{n}\left(\mathrm{i} \cos (n \phi)-\frac{\kappa n}{C} \sin (n \phi)\right) & \text { If } n \text { is odd }
\end{array} .\right.
\end{aligned}
$$

The $R$ integral is of the form

$$
I_{n o}[f]\left(s, R^{\prime}\right)=\int_{0}^{R^{\prime}} \mathrm{d} R R f(R, s) J_{n}(C(s) R) .
$$

General analytic results exist for particular forms of $f$ (see [21] for example), notably when $f$ is a Gaussian. We define a function $\lambda(n, s)$ as

$$
\begin{gathered}
\lambda_{n}(s)= \\
(-1)^{n} \sqrt{2 \pi}\left\{\begin{array}{cc}
\Phi_{1}(s) I_{n o}\left[a_{n}\right](s, 1 / \kappa)+\Phi_{2}(s) I_{n o}\left[b_{n}\right](s, 1 / \kappa) & \text { If } n \text { is even, } \\
-i \Phi_{2}(s) I_{n o}\left[a_{n}\right](s, 1 / \kappa)+i \Phi_{1}(s) I_{n o}\left[b_{n}\right](s, 1 / \kappa) & \text { If } n \text { is odd, } \\
\Phi_{1}=\left(\cos (n \phi)+\frac{i \kappa n}{C} \sin (n \phi)\right) & \\
\Phi_{2}=\left(-\sin (n \phi)+\frac{i \kappa n}{C} \cos (n \phi)\right) .
\end{array}\right.
\end{gathered}
$$


Note that the functions $\Phi_{1 / 2}$ express the effect of the additional rotation $\phi$ on the transform. Finally we can express $F_{\text {no }}$ as

$$
F_{\text {no }}\left[\rho, \mathcal{R} \mathbf{y}+\mathbf{x}_{\mathrm{c}}, R_{m}\right](\mathbf{k})=\mathrm{e}^{\mathrm{i} \mathbf{k} \cdot \mathbf{x}_{\mathrm{c}}} \sum_{n=0}^{\infty} \int_{0}^{L} \mathrm{~d} s \lambda_{n} \mathrm{e}^{\mathrm{ik} \cdot \mathcal{R} \mathbf{r}} .
$$

4.1.1. The axially symmetric case In the case in which $\rho$ has no dependence on $\theta$ we only retain the $n=0$ terms form the Fourier series. Writing $a_{0}(s, R)=\rho_{\text {as }}(s, R)$ (axially symmetric), we have

$$
F_{\text {no }}\left[\rho_{\text {as }}, \mathcal{R} \mathbf{y}+\mathbf{x}_{\mathrm{c}}, R_{m}\right](\mathbf{k})=\mathrm{e}^{\mathrm{i} \mathbf{k} \cdot \mathbf{x}_{\mathrm{c}}} \int_{0}^{L} \mathrm{~d} s \lambda_{0} \mathrm{e}^{\mathrm{i} \mathbf{k} \cdot \mathcal{R} \mathbf{r}} .
$$

4.1.2. Constant density and restricted radius We can obtain a simple result if the density function has a constant value $\left(\rho_{c}=D_{c}\right.$, with $D_{c}$ constant). Using the result [21],

$$
\int_{0}^{R_{\mathrm{m}}} \mathrm{d} R R J_{0}(C R)=\frac{R_{\mathrm{m}} J_{1}\left(C R_{\mathrm{m}}\right)}{C},
$$

and assuming $R_{\mathrm{m}}$ is such that $R_{\mathrm{m}}<1 / \kappa(s), \forall s$, we have

$F\left[\rho_{\mathrm{c}}, R \mathbf{y}+\mathbf{x}_{\mathrm{c}}\right](\mathbf{k})=F_{\mathrm{no}}\left[\rho_{\mathrm{c}}, R \mathbf{y}+\mathbf{x}_{\mathrm{c}}\right](\mathbf{k})=\frac{D_{c} R_{\mathrm{m}} \mathrm{e}^{\mathrm{i} \mathbf{k} \cdot \mathbf{x}_{\mathrm{c}}}}{\sqrt{2 \pi}} \int_{0}^{L} \mathrm{~d} s \frac{J_{1}\left(C R_{m}\right) \mathrm{e}^{\mathrm{i} \mathbf{k} \cdot \mathcal{R} \mathbf{r}}}{C}$.

4.2. Tubes with local overlap $R>1 / \kappa$

Next we need to evaluate the contribution of the density on the subset of the manifold on which there is local overlap. That is, for values $R \geq 1 / \kappa$. The substitutions (18) are not affected by the modulus sign, applying them we have

$F_{\mathrm{o}}\left[\rho, \mathcal{R} \mathbf{y}+\mathbf{x}_{\mathrm{c}}, R_{m}\right](\mathbf{k})$

$=\frac{\mathrm{e}^{\mathrm{i} \mathbf{k} \cdot \mathbf{x}_{\mathrm{c}}}}{\sqrt{2 \pi}} \sum_{n=0}^{\infty}(-1)^{n} \int_{0}^{L} \mathrm{~d} s \mathrm{e}^{\mathrm{i} \mathbf{k} \cdot \mathcal{R} \mathbf{r}} \int_{1 / \kappa}^{\infty} \mathrm{d} R R$

$\int_{-\pi}^{\pi} \mathrm{d} \theta\left(a_{n} \cos (n(\theta-\phi))+b_{n} \sin (n(\theta-\phi))|1+\kappa R \cos \theta| \mathrm{e}^{\mathrm{i} R C \sin \theta}\right.$.

We have also used the integrand's periodicity to alter the range of integration as in the previous section. The $\theta$ integrand must be split into two parts as the Jacobian becomes negative for some $\theta$ subsets of $S^{1}$. In particular $1+\kappa R \cos (\theta)$ is 0 on $\theta \in[-\pi, \pi]$ when

$$
\begin{aligned}
& \theta= \pm \theta_{m} \\
& \theta_{m}=\cos ^{-1}\left(-\frac{1}{R \kappa}\right),
\end{aligned}
$$


which is well-defined as $-1 /(R \kappa)$ is restricted to the range $[0,-1]$ for $R \geq 1 / \kappa$. Using this knowledge (29) can be written as

$F_{\mathrm{o}}\left[\rho, \mathcal{R} \mathbf{y}+\mathbf{x}_{\mathrm{c}}, R_{m}\right](\mathbf{k})$

$=\frac{\mathrm{e}^{\mathrm{i} \mathbf{k} \cdot \mathbf{x}_{\mathrm{c}}}}{\sqrt{2 \pi}} \sum_{n=0}^{\infty}(-1)^{n} \int_{0}^{L} \mathrm{~d} s \mathrm{e}^{\mathrm{i} \mathbf{k} \cdot \mathcal{R} \mathbf{r}} \int_{1 / \kappa}^{\infty} \mathrm{d} R R\left[a_{n}\left(\Theta_{\mathrm{C}, n}^{\mathrm{P}}-\Theta_{\mathrm{C}, n}^{N}\right)+b_{n}\left(\Theta_{\mathrm{S}, n}^{\mathrm{P}}-\Theta_{\mathrm{S}, n}^{N}\right)\right]$.

Where $\Theta_{\mathrm{C} / \mathrm{S}, n}^{\mathrm{P}}(s, R)$ are bounded functions representing the $n^{\text {th }}$ contribution to the the total $\theta$ integral on the domain $\theta \in\left[-\theta_{m}, \theta_{m}\right]$, for which $\kappa R \cos \theta \geq-1$. $\Theta_{\mathrm{C} / \mathrm{S}, n}^{N}(s, R)$ are bounded functions representing the $n^{\text {th }}$ contribution to the the total $\theta$ integral on the domains $\theta \in\left[-\pi,-\theta_{m}\right)$ and $\theta \in\left(\theta_{m}, \pi\right]$, for which $\kappa R \cos \theta<-1$. The functions $\Theta_{\mathrm{C} / \mathrm{S}, n}^{P}(s, R)$ are

$$
\begin{aligned}
& \Theta_{\mathrm{C}, n}^{\mathrm{P}}(s, R)=\int_{-\theta_{m}}^{\theta_{m}} \cos (n(\theta-\phi))(1+\kappa R \cos \theta) \mathrm{e}^{\mathrm{i} R C \sin \theta} \mathrm{d} \theta, \\
& \Theta_{\mathrm{S}, n}^{\mathrm{P}}(s, R)=\int_{-\theta_{m}}^{\theta_{m}} \sin (n(\theta-\phi))(1+\kappa R \cos \theta) \mathrm{e}^{\mathrm{i} R C \sin \theta} \mathrm{d} \theta .
\end{aligned}
$$

The functions $\Theta_{C / S, n}^{\mathrm{N}}(s, R)$ are

$\Theta_{\mathrm{C}, n}^{\mathrm{N}}(s, R)$

$=\int_{-\pi}^{-\theta_{m}} \cos (n(\theta-\phi))(1+\kappa R \cos \theta) \mathrm{e}^{\mathrm{i} R C \sin \theta} \mathrm{d} \theta+\int_{\theta_{m}}^{\pi} \cos (n(\theta-\phi))(1+\kappa R \cos \theta) \mathrm{e}^{\mathrm{i} R C \sin \theta} \mathrm{d} \theta$, $\Theta_{\mathrm{S}, n}^{\mathrm{N}}(s, R)$

$=\int_{-\pi}^{-\theta_{m}} \sin (n(\theta-\phi))(1+\kappa R \cos \theta) \mathrm{e}^{\mathrm{i} R C \sin \theta} \mathrm{d} \theta+\int_{\theta_{m}}^{\pi} \sin (n(\theta-\phi))(1+\kappa R \cos \theta) \mathrm{e}^{\mathrm{i} R C \sin \theta} \mathrm{d} \theta$.

Finally, we define

$$
\eta_{n}(s)=\frac{(-1)^{n}}{\sqrt{2 \pi}} \int_{1 / \kappa}^{\infty} \mathrm{d} R R\left[a_{n}\left(\Theta_{\mathrm{C}, n}^{\mathrm{P}}-\Theta_{\mathrm{C}, n}^{\mathrm{N}}\right)+b_{n}\left(\Theta_{\mathrm{S}, n}^{P}-\Theta_{\mathrm{S}, n}^{N}\right)\right]
$$

giving

$$
F_{\mathrm{o}}\left[\rho, \mathcal{R} \mathbf{y}+\mathbf{x}_{\mathrm{c}}, R_{m}\right](\mathbf{k})=\mathrm{e}^{\mathrm{i} \mathbf{k} \cdot \mathbf{x}_{\mathrm{c}}} \sum_{n=0}^{\infty} \int_{0}^{L} \mathrm{~d} s \eta_{n} \mathrm{e}^{\mathrm{i} \mathbf{k} \cdot \mathcal{R} \mathbf{r}} .
$$

For an axially symmetric density we restrict to the $n=0$ part of the sum, as in the previous section.

\subsection{The general tube-density transform}

We can now write the full Fourier transform as

$$
F\left[\rho, R \mathbf{y}+\mathbf{x}_{\mathrm{c}}, R_{m}\right](\mathbf{k})=F_{\text {no }}+F_{\mathrm{o}}=\mathrm{e}^{\mathrm{i} \mathbf{k} \cdot \mathbf{x}_{\mathrm{c}}} \sum_{n=0}^{\infty} \int_{0}^{L} \mathrm{~d} s\left(\lambda_{n}+\eta_{n}\right) \mathrm{e}^{\mathrm{i} \mathbf{k} \cdot \mathcal{R} \mathbf{r}} .
$$


Where $\lambda_{n}$ represents the contributions of a subset of the manifold for which there is no local overlap $(R<1 / \kappa)$. It is defined using (23) in conjunction with (22). The function $\eta_{n}$ is the contribution of the complement of this subset $(R \geq 1 / \kappa(s))$. It is defined using equation (36) in conjunction with equations (30), (32), (33), (34) and (35). We recover the axially symmetric expression for both $\lambda$ and $\eta$ by considering only the $n=0$ contributions.

The evaluation of $F_{\text {no }}$ is relatively simple, especially in the case in which (22) has anti-derivative results, for which it reduces to an arclength integral. The evaluation of $F_{\text {no }}$, however, will generally require a numerical integration over all three parameters.

\subsection{The transform as an ordinary differential equation}

In the case in which the tube's maximum radius $R_{\mathrm{m}}<1 / \kappa, \forall s$, we only need to evaluate

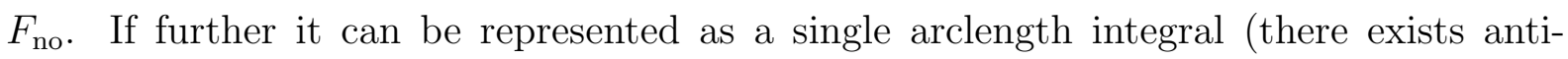
derivative results for $(22)$ ), we can differentiate $F$ with respect to $s$ to create a differential equation

$$
F^{\prime}\left[\rho, R \mathbf{y}+\mathbf{x}_{\mathrm{c}}, R_{m}\right](\mathbf{k})=\mathrm{e}^{\mathrm{i} \mathbf{k} \cdot \mathbf{x}_{\mathrm{c}}} \sum_{i=1}^{n} \lambda_{n} \mathrm{e}^{\mathrm{i} \mathbf{k} \cdot\left(\mathcal{R} \mathbf{r}+\mathbf{x}_{\mathrm{c}}\right)} .
$$

If the function set $\left(u_{1}, u_{2}, u_{3}\right)$ are specified we can use this equation to augment the Darboux system (3) and generate $F$. That is, for a given $\mathbf{k}$, the Fourier transform is obtained by a numerical integration of a system of nonlinear differential equations for $s$ from 0 to $L$.

\subsection{The general surface transform}

If we are interested in a particular tubular surface of radius $R_{s}$ then we can evaluate the transform of the density on that particular surface as follows. Inserting (13) into (11) (and taking into account an affine map as in (14)) we have

$F\left[\rho, \mathcal{R} \mathbf{y}+\mathbf{x}_{\mathrm{c}}, R_{s}\right](\mathbf{k})=\frac{\mathrm{e}^{\mathrm{i} \mathbf{k} \cdot \mathbf{x}_{\mathrm{c}}}}{\sqrt{2 \pi}} \int_{0}^{L} \mathrm{~d} s \mathrm{e}^{\mathrm{i} \mathbf{k} \cdot \mathcal{R} \mathbf{r}} \int_{0}^{2 \pi} \mathrm{d} \theta \rho|J| \mathrm{e}^{\mathrm{i} R_{s} \mathbf{k} \cdot \mathcal{R}\left(\mathbf{d}_{1} \cos \theta+\mathbf{d}_{2} \sin \theta\right)}$.

\subsection{Case 1 - No local overlap}

If $R_{\mathrm{s}}<1 / \kappa(s), \forall s$, the surface has no local overlap. We use the Fourier series expansion of $\rho\left(s, R_{\mathrm{s}}, \theta\right)$ (17) and make the substitutions (18). Following the same procedure as in Sections 4.1 and Appendix A, we obtain the following result

$$
\begin{aligned}
& F\left[\rho, \mathcal{R} \mathbf{y}+\mathbf{x}_{\mathrm{c}}, R_{s}\right](\mathbf{k})=\sqrt{2 \pi} R_{s} \mathrm{e}^{\mathrm{i} \mathbf{k} \cdot \mathbf{x}_{\mathrm{c}}} \sum_{n=0}^{\infty}(-1)^{n} \int_{0}^{L} \mathrm{~d} s \mathrm{e}^{\mathrm{i} \mathbf{k} \cdot \mathcal{R} \mathbf{r}} \\
& J_{n}\left(C R_{s}\right)\left\{\begin{array}{cc}
a_{n}\left(\cos (n \phi)+\frac{\mathrm{i} \kappa n}{C} \sin (n \phi)\right)+b_{n}\left(-\sin (n \phi)+\frac{\mathrm{i} \kappa n}{C} \cos (n \phi)\right) & \text { If } n \text { is even } \\
a_{n}\left(\mathrm{i} \sin (n \phi)+\frac{\kappa n}{C} \cos (n \phi)\right)+b_{n}\left(\mathrm{i} \cos (n \phi)-\frac{\kappa n}{C} \sin (n \phi)\right) & \text { If } n \text { is odd }
\end{array}\right.
\end{aligned}
$$


In the axially symmetric case this reduces to

$$
F\left[\rho_{\text {as }}, \mathcal{R} \mathbf{y}+\mathbf{x}_{\mathrm{c}}, R_{s}\right](\mathbf{k})=\sqrt{2 \pi} R_{s} \mathrm{e}^{\mathrm{i} \mathbf{k} \cdot \mathbf{x}_{\mathrm{c}}} \int_{0}^{L} \mathrm{~d} s \rho_{\text {as }} J_{0}\left(C R_{s}\right) \mathrm{e}^{\mathrm{i} \mathbf{k} \cdot \mathcal{R} \mathbf{r}} .
$$

\subsection{Case 2 - local overlap}

For $R_{\mathrm{s}}>1 / \kappa(s), \forall s$ we have

$F\left[\rho, \mathcal{R} \mathbf{y}+\mathbf{x}_{\mathrm{c}}, R_{s}\right](\mathbf{k})$

$=\frac{R_{\mathrm{s}} \mathrm{e}^{\mathrm{i} \mathbf{k} \cdot \mathbf{x}_{\mathrm{c}}}}{\sqrt{2 \pi}} \sum_{n=0}^{\infty}(-1)^{n} \int_{0}^{L} \mathrm{~d} s \mathrm{e}^{\mathrm{i} \mathbf{k} \cdot \mathcal{R} \mathbf{r}}\left[a_{n}\left(\Theta_{\mathrm{C}, n}^{\mathrm{P}}-\Theta_{\mathrm{C}, n}^{\mathrm{N}}\right)+b_{n}\left(\Theta_{\mathrm{S}, n}^{\mathrm{P}}-\Theta_{\mathrm{S}, n}^{\mathrm{N}}\right)\right]$,

where the $\Theta$ functions are defined by (30), (32), (33), (34) and (35). In the axially symmetric case we have

$$
F\left[\rho_{\mathrm{as}}, \mathcal{R} \mathbf{y}+\mathbf{x}_{\mathrm{c}}, R_{s}\right](\mathbf{k})=\frac{R_{s} \mathrm{e}^{\mathrm{i} \mathbf{k} \cdot \mathbf{x}_{\mathrm{c}}}}{\sqrt{2 \pi}} \int_{0}^{L} \mathrm{~d} s \rho_{\mathrm{as}}\left(\Theta_{\mathrm{C}, 0}^{\mathrm{P}}-\Theta_{\mathrm{C}, 0}^{\mathrm{N}}\right) \mathrm{e}^{\mathrm{i} \mathbf{k} \cdot \mathcal{R} \mathbf{r}} .
$$

\section{Restricting the $\eta$ contribution}

We now consider radially unbounded manifolds. In what follows we derive conditions on the radial rate of decay of $\rho$ such that the contribution to (38) from $\eta(s)$ is negligible. This allows us to approximate the full Fourier transform with the much simpler $F_{\text {no }}$ expression. In particular we establish conditions for which the contribution of $F_{\mathrm{o}}$ is $\mathcal{O}(\epsilon)$ compared to $F_{\text {no }}$, with $\epsilon$ a positive constant which can be made arbitrarily small.

To do this we compare the relative size of the $R$ integrals on the domains $R \in[0,1 / \kappa)$ and $R \in[1 / \kappa, \infty]$. In order to make a direct comparison we must first compare the $\theta$ integrals on the two domains, since they can be viewed as functions of $R$. We can split the $\theta$ integrals on the domain $R \in[0,1 / \kappa)$ into the same set of $\theta$ domains as we did in (31). That is, we have

$$
\lambda_{n}(s)=\int_{0}^{1 / \kappa} \mathrm{d} R R\left[a_{n}\left(\Theta_{\mathrm{C}, n}^{\mathrm{P}}+\Theta_{\mathrm{C}, n}^{\mathrm{N}}\right)+b_{n}\left(\Theta_{\mathrm{S}, n}^{\mathrm{P}}+\Theta_{\mathrm{S}, n}^{\mathrm{N}}\right)\right],
$$

which can be compared to $\eta_{n}(s)$. The only difference is that we do not have a factor $(-1)$ on the domains $|\theta|>\theta_{m}$ as $\kappa R \cos \theta>-1, \forall \theta$. The $\Theta$ functions require the evaluation of integrals of the following form

$$
\begin{aligned}
& \int_{a}^{b} \mathrm{~d} \theta \cos (n(\theta-\phi)) \mathrm{e}^{\mathrm{i} R C \sin \theta}+\kappa R \int_{a}^{b} \mathrm{~d} \theta \cos (\theta) \cos (n(\theta-\phi)) \mathrm{e}^{\mathrm{i} R C \sin \theta}, \\
& \int_{a}^{b} \mathrm{~d} \theta \sin (n(\theta-\phi)) \mathrm{e}^{\mathrm{i} R C \sin \theta}+\kappa R \int_{a}^{b} \mathrm{~d} \theta \cos (\theta) \cos (n(\theta-\phi)) \mathrm{e}^{\mathrm{i} R C \sin \theta},
\end{aligned}
$$

where $[a, b] \subset[-\pi, \pi]$. The individual integrals (not including the pre multiplier $\kappa R$ ) are bounded in magnitude by $2 \pi$ as they are composed of trigonometric functions. So the 

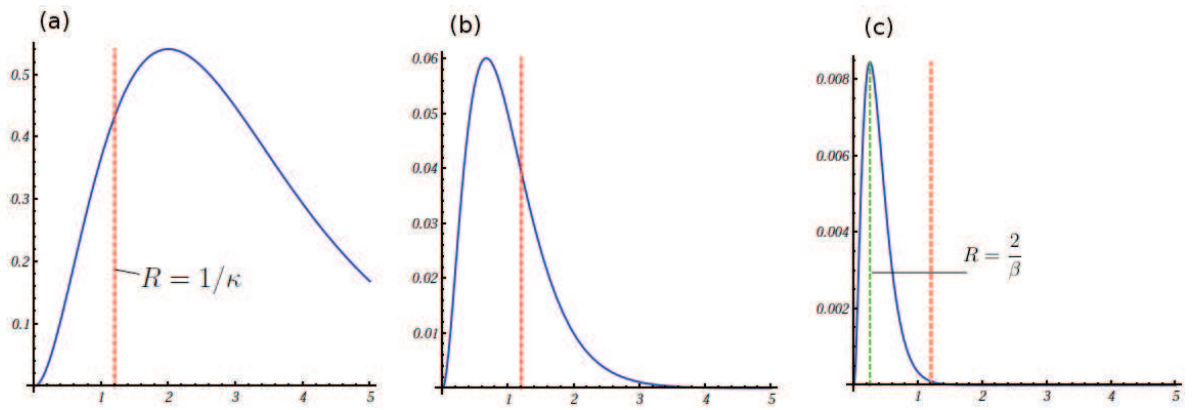

Figure 7. Representative plots of the integrand $R^{2} \exp (-\beta R)$. In (a) the integrands maximum is for a value $R>1 / \kappa$ and the inequalities (48) cannot be satisfied. In (b) the maximum is to the left of $1 / \kappa$ but the decay rate of the function would not be sufficient for (48) to be satisfied. In (c) the rate of decay is sufficient for (48) to be satisfied.

upper bound on the difference between the $\theta$ part of $\lambda$ and $\eta$ is determined by $R$ ( $\kappa$ being the same for both). We can take advantage of this for a particular form $f\left(s^{\prime}, R\right)$ of the radial dependence of $a_{n}$ or $b_{n}$, at an arclength $s^{\prime}$, by requiring the following inequalities are satisfied

$$
\frac{\int_{1 / \kappa}^{\infty} \mathrm{d} R R f\left(s^{\prime}, R\right)}{\int_{0}^{1 / \kappa} \mathrm{d} R R f\left(s^{\prime}, R\right)} \leq \epsilon, \quad \frac{\int_{1 / \kappa}^{\infty} \mathrm{d} R R^{2} f\left(s^{\prime}, R\right)}{\int_{0}^{1 / \kappa} \mathrm{d} R R^{2} f\left(s^{\prime}, R\right)} \leq \epsilon
$$

If the inequalities (48) hold for all $a_{n}$ and $b_{n}$ then the function $\eta\left(s^{\prime}\right)$ is $\mathcal{O}(\epsilon)$ in comparison to $\lambda\left(s^{\prime}\right)$ at $s^{\prime}$. If further this holds for each $s^{\prime} \in[0, L]$ then we have that $F_{\mathrm{o}}$ is $\mathcal{O}(\epsilon)$ in comparison to $F_{\text {no. }}$.

We can obtain bounds on the integrals of (48) by considering a function decaying exponentially with $R$. In particular we consider the function $\exp (-\beta(s) R)$, with $\beta(s)>0$ controlling the rate of decay and $A$ the maximum density value. We shall derive an inequality relating $\beta$ to the axis curvature $\kappa$. The integrals will be labelled $I_{1}^{R}$ and $I_{1}^{R^{2}}$ on $R \in[0,1 / \kappa]$ and $I_{2}^{R}, I_{2}^{R^{2}}$ on $R \in[1 / \kappa, \infty]$. We have

$I_{2}^{R}=\int_{\frac{1}{\kappa}}^{\infty} \mathrm{d} R R A \mathrm{e}^{-\beta R}=-\frac{A}{\beta}\left[R \mathrm{e}^{-\beta R}\right]_{\frac{1}{\kappa}}^{\infty}+\frac{A}{\beta} \int_{\frac{1}{\kappa}}^{\infty} \mathrm{d} R \mathrm{e}^{-\beta R}=A \mathrm{e}^{-\beta / \kappa}\left[\frac{\kappa^{-1}}{\beta}+\frac{1}{\beta^{2}}\right]$.

In order that (48) can hold the integrands $R \exp (-\beta R)$ and $R^{2} \exp (-\beta R)$ will have a single maximum on the domain $R \in[0,1 / \kappa)$ (see Figure 7 ). Also they will be zero at $R=0$. Thus we can create a lower bound for the integrals on this domain using by approximating them from below as a triangle, with one vertex at the origin and one at the coordinates $\left(\kappa^{-1}, A \kappa^{-1} \exp (-\beta / \kappa)\right)$ or $\left(\kappa^{-1}, A \kappa^{-2} \exp (-\beta / \kappa)\right)$. We have

$$
I_{1}^{R}>\frac{A \mathrm{e}^{-\beta / \kappa} \kappa^{-2}}{2} .
$$


The ratio $I_{2}^{R} / I_{1}^{R}$ satisfies

$$
\frac{I_{2}^{R}}{I_{1}^{R}}<\frac{2 \kappa^{-1} \beta+1}{\beta^{2} \kappa^{-2}}<\frac{2}{\beta \kappa^{-1}},
$$

remembering $R, \kappa>0$. In turn we require the R.H.S of (51) be less than or equal to $\epsilon$, from which we obtain

$$
\beta \geq \frac{2 \kappa}{\epsilon} .
$$

We now show this rate will also ensure the inequalities (48) are satisfied for the integrand $R^{2} \exp (-\beta R)$. We have

$$
\begin{aligned}
& I_{2}^{R^{2}}=\int_{\frac{1}{\kappa}}^{\infty} \mathrm{d} R R R^{2} A \mathrm{e}^{-\beta R}=-\frac{1}{\beta}\left[A \mathrm{e}^{-\beta R} R^{2}\right]+\frac{2 A}{\beta} \int_{1 / \kappa}^{\infty} \mathrm{e}^{-\beta R} R \mathrm{~d} R \\
&=A \mathrm{e}^{-\beta / \kappa}\left[\frac{\kappa^{-2}}{\beta}+\frac{2}{\beta}\left[\frac{\kappa^{-1}}{\beta}+\frac{1}{\beta^{2}}\right]\right]=\frac{A \mathrm{e}^{-\beta / \kappa}}{\beta^{3}}\left[\kappa^{-2} \beta^{2}+2 \beta \kappa^{-1}+2\right] .
\end{aligned}
$$

The integral on $[0,1 / \kappa]$ can be bounded from below using a triangular area

$$
I_{1}^{R^{2}}>\frac{A \mathrm{e}^{-\beta / \kappa} \kappa^{-3}}{2}
$$

Thus we have

$$
\frac{I_{2}^{R^{2}}}{I_{1}^{R^{2}}}<\frac{2\left(\kappa^{-2} \beta^{2}+2 \beta \kappa^{-1}+2\right)}{\beta^{3} \kappa^{-3}}<\frac{2}{\beta \kappa^{-1}} .
$$

Substituting $\beta=2 \kappa / \epsilon$ will yield the required inequality. So, if $\beta \geq 2 \kappa / \epsilon$ the inequalities (48) hold. We can use this estimation as a basis for comparing other choices of $f(s, R)$. If the following inequalities hold

$$
\begin{aligned}
& \int_{1 / \kappa}^{\infty} f\left(s^{\prime}, R\right) R \mathrm{~d} R<\frac{A \mathrm{e}^{-2 / \epsilon}}{4 \kappa^{2}}\left[2 \epsilon+\epsilon^{2}\right], \\
& \int_{1 / \kappa}^{\infty} f\left(s^{\prime}, R\right) R^{2} \mathrm{~d} R<\frac{A \mathrm{e}^{-2 / \epsilon}}{8 \kappa^{3}}\left[4 \epsilon+4 \epsilon^{2}+2 \epsilon^{3}\right], \\
& \int_{0}^{\frac{1}{\kappa}} f\left(s^{\prime}, R\right) R \mathrm{~d} R>\frac{A \mathrm{e}^{-2 / \epsilon} \kappa^{-2}}{2}, \\
& \int_{0}^{\frac{1}{\kappa}} f\left(s^{\prime}, R\right) R^{2} \mathrm{~d} R>\frac{A \mathrm{e}^{-2 / \epsilon} \kappa^{-3}}{2} .
\end{aligned}
$$

then (48) are satisfied at $s=s^{\prime}$. The estimations are determined by the curvature of the tube's axis. As expected, the larger the curvature the faster the required rate of decay.

Given the inequalities (48) are satisfied for the whole manifold we have the approximation $F(\mathbf{k}) \approx F_{\text {no }}(\mathbf{k})$, with the quality of the approximation determined by the value of $\epsilon$. In addition, within the same approximation, we can extend the domain of integration of $R$ for $(27)$ to $R \in[0, \infty]$. In this case the $R$ integral is the Hankel transform, for which there are a number of known analytic results, see for example [21]. 


\section{Conclusions}

We have constructed a model for a continuum tubular density. The density is defined by specifying the geometry of the axis curve $\mathbf{r}$, and a vector field $\mathbf{d}_{1}$ which determines whether the manifold has twisted structure. Points on the manifold are specified by the triple $(s, R, \theta)$, with $s$ arclength, $R$ the tube radius and $\theta$ the point on a circle of the tube surface for fixed $s$ and $R$ values (Section 2). A continuous scalar density function is then defined. This density can represent a physical quantity such as an electron density or volume of magnetic flux. A tempered mapping is used to represent the density distribution (Section 3). Where there is density overlap the net density is calculated using the superposition principle. In order to take advantage of tubular forms constructed from an ensemble of repeated structures, such as those encountered for protein tertiary and quaternary structures, we extend our density definition to include an arbitrary affine transform (Section 3.3).

By representing the density function $\rho$ as a Fourier series we derive the most general expression of the Fourier transform of this density (Section 4.3). In doing so we handle existence of density overlap by splitting the integral into two components. One for a subset of the manifold whose radius is less than the critical curvature condition, $R<\kappa$, on this set the tube's Jacobian is smooth. For the complement of this subset $(R \geq \kappa)$ we ensure the Jacobian is positive definite by splitting the $\theta$ integral. In doing so we ensure the net density is always correctly evaluated. Particular cases of an axially symmetric density (Section 4.3) and a constant density (Section 4.1.2) are detailed. In Section 5 we define conditions on the radial rate of decay of the density, such that the full transform can be approximated by only the contribution for which $R<1 / \kappa$. This greatly simplifies the evaluation of the transform. In particular specific forms of the density function can be evaluated as a single arclength integral. The required conditions are (48). A set of upper and lower bounds on the integrals in the inequalities (48) were calculated based on an exponential decay rate. The estimates are determined by the curvature of the axis $\kappa$. Finally, we detail expressions for the Fourier transform of a single tubular surface of the manifold in Section 4.5.

\section{Appendix A. Evaluation of the $\theta$ integrals for $F_{\text {no }}$}

In this section we demonstrate that each $\theta$ integral (19) and (20) can be written in terms of a Bessel function.

As the integrands (19) and (20) are periodic in $\theta$ we can change the range of integration to $\theta \in[-\pi, \pi]$. Thus, we must evaluate the following integrals

$$
\Theta_{\mathrm{C} \kappa^{0}}=\left(a_{n} \cos (n \phi)-b_{n} \sin (n \phi)\right) \int_{-\pi}^{\pi} \cos (n \theta) \mathrm{e}^{\mathrm{i} R C \sin \theta} \mathrm{d} \theta,
$$




$$
\begin{aligned}
& \Theta_{\mathrm{C} \kappa}=\kappa R\left(a_{n} \cos (n \phi)-b_{n} \sin (n \phi)\right) \int_{-\pi}^{\pi} \cos (n \theta) \cos (\theta) \mathrm{e}^{\mathrm{i} R C \sin \theta} \mathrm{d} \theta \\
& \Theta_{\mathrm{S} \kappa^{0}}=\left(a_{n} \sin (n \phi)+b_{n} \cos (n \phi)\right) \int_{-\pi}^{\pi} \sin (n \theta) \mathrm{e}^{\mathrm{i} R C \sin \theta} \mathrm{d} \theta, \\
& \Theta_{\mathrm{S} \kappa}=\kappa R\left(a_{n} \cos (n \phi)+b_{n} \sin (n \phi)\right) \int_{-\pi}^{\pi} \sin (n \theta) \cos (\theta) \mathrm{e}^{\mathrm{i} R C \sin \theta} \mathrm{d} \theta,
\end{aligned}
$$

We first deal with $\Theta_{(\mathrm{C} / \mathrm{S}) \kappa^{0}}$ contribution. We use the complex exponential form of cos and sin to write the integrals as

$$
\begin{aligned}
& \frac{1}{2}\left[\int_{-\pi}^{\pi} \mathrm{d} \theta \mathrm{e}^{-\mathrm{i}(-n \theta-R C \sin \theta)}+\int_{-\pi}^{\pi} \mathrm{d} \theta \mathrm{e}^{-\mathrm{i}(n \theta-R C \sin \theta)}\right], \\
& \frac{1}{2 i}\left[\int_{-\pi}^{\pi} \mathrm{d} \theta \mathrm{e}^{-\mathrm{i}(-n \theta-R C \sin \theta)}-\int_{-\pi}^{\pi} \mathrm{d} \theta \mathrm{e}^{-\mathrm{i}(n \theta-R C \sin \theta)}\right] .
\end{aligned}
$$

We recognise these functions as being, up to a constant multiplier, the integral definition of Bessel's functions $\left(J_{n}\right)$ of the first kind

$$
J_{n}(z)=\frac{1}{2 \pi} \int_{-\pi}^{\pi} \mathrm{d} \tau \mathrm{e}^{-\mathrm{i}(n \tau-z \sin \tau)},
$$

where $n$ is the order of the function and $z$ is either real or complex (here $R C$ is always real). Thus we can write equations (A.1) and (A.2) as

$$
\begin{aligned}
& \Theta_{\mathrm{C} \kappa^{0}}=\left(a_{n} \cos (n \phi)-b_{n} \sin (n \phi)\right) \pi\left[J_{-n}(R C)+J_{n}(R C)\right], \\
& \Theta_{\mathrm{S} \kappa^{0}}=-\left(a_{n} \sin (n \phi)+b_{n} \cos (n \phi)\right) \pi i\left[J_{-n}(R C)-J_{n}(R C)\right] .
\end{aligned}
$$

Applying the Bessel function identity $J_{-n}(z)=(-1)^{n} J_{n}(z)$ we have

$$
\begin{aligned}
& \Theta_{\mathrm{C} \kappa^{0}}=\left(a_{n} \cos (n \phi)-b_{n} \sin (n \phi)\right)\left\{\begin{array}{cc}
2 \pi J_{n}(R C) & \text { If } n \text { is even } \\
0 & \text { If } n \text { is odd }
\end{array},\right. \\
& \Theta_{\mathrm{S} \kappa^{0}}=\left(a_{n} \sin (n \phi)+b_{n} \cos (n \phi)\right)\left\{\begin{array}{cc}
0 & \text { If } n \text { is even } \\
2 \pi i J_{n}(R C) & \text { If } n \text { is odd }
\end{array} .\right.
\end{aligned}
$$

Now we consider $\Theta_{(C / S) \kappa}$. We use standard trigonometric identities to write these integrals as

$$
\begin{aligned}
\Theta_{\mathrm{C} \kappa} & =\frac{\kappa R}{2} \int_{-\pi}^{\pi}[\cos ((n+1) \theta)+\cos ((n-1) \theta)] \mathrm{e}^{\mathrm{i} R C \sin \theta} \mathrm{d} \theta, \\
\Theta_{\mathrm{S} \kappa} & =\frac{\kappa R}{2} \int_{-\pi}^{\pi}[\sin ((n+1) \theta)+\sin ((n-1) \theta)] \mathrm{e}^{\mathrm{i} R C \sin \theta} \mathrm{d} \theta .
\end{aligned}
$$

Inserting the complex exponential forms as in equations (A.5) and (A.6), and using the Bessel integral identity (A.7) this becomes

$$
\begin{aligned}
& \Theta_{\mathrm{C} \kappa}=\frac{\pi \kappa R}{2}\left\{\left[J_{-(n+1)}(R C)+J_{n+1}(R C)\right]+\left[J_{-(n-1)}(R C)+J_{n-1}(R C)\right]\right\}, \\
& \Theta_{\mathrm{S} \kappa}=\frac{-\mathrm{i} \pi \kappa R}{2}\left\{\left[J_{-(n+1)}(R C)-J_{n+1}(R C)\right]+\left[J_{-(n-1)}(R C)-J_{n-1}(R C)\right]\right\},
\end{aligned}
$$


Which becomes

$$
\begin{aligned}
& \Theta_{\mathrm{C} \kappa}=\left\{\begin{array}{cc}
0 & \text { If } n \text { is even } \\
\pi \kappa R\left[J_{n+1}(R C)+J_{n-1}(R C)\right] & \text { If } n \text { is odd }
\end{array},\right. \\
& \Theta_{\mathrm{S} \kappa}=\left\{\begin{array}{cc}
\mathrm{i} \pi \kappa R\left[J_{n+1}(R C)+J_{n-1}(R C)\right] & \text { If } n \text { is even, } \\
0 & \text { If } n \text { is odd }
\end{array} .\right.
\end{aligned}
$$

Finally, we apply the following Bessel identity

$$
\frac{2 \alpha}{z} J_{\alpha}(z)=J_{\alpha+1}(z)+J_{\alpha-1}(z)
$$

to obtain

$$
\begin{aligned}
& \Theta_{\mathrm{C} \kappa}=\left(a_{n} \cos (n \phi)-b_{n} \sin (n \phi)\right)\left\{\begin{array}{cc}
0 & \text { If } n \text { is even } \\
\frac{2 n \pi \kappa}{C} J_{n}(R C) & \text { If } n \text { is odd }
\end{array}\right. \\
& \Theta_{\mathrm{S} \kappa}=\left(a_{n} \sin (n \phi)+b_{n} \cos (n \phi)\right)\left\{\begin{array}{cc}
\frac{2 n i \pi \kappa}{C} J_{n}(R C) & \text { If } n \text { is even, } \\
0 & \text { If } n \text { is odd }
\end{array} .\right.
\end{aligned}
$$

The total transform can be written as

$$
F_{\text {no }}=\frac{\mathrm{e}^{\mathrm{i} \mathbf{k} \cdot \mathbf{x}_{\mathrm{c}}}}{\sqrt{2 \pi}} \sum_{i=0}^{n}(-1)^{n} \int_{0}^{L} \mathrm{~d} s \mathrm{e}^{\mathrm{ik} \cdot \mathcal{R} \mathbf{r}} \int_{0}^{1 / \kappa} \mathrm{d} R R\left(\Theta_{\mathrm{C} \kappa}+\Theta_{\mathrm{C} \kappa}{ }^{0}+\Theta_{\mathrm{S} \kappa}+\Theta_{\mathrm{S} \kappa^{0}}\right) .
$$

Inserting (A.8),(A.9), (A.16) and (A.17) into (A.18) gives (21).

\section{Acknowledgements}

This publication is based on work supported by Award No. KUK-C1-013-04, made by King Abdullah University of Science and Technology (KAUST). AG is a Wolfson Royal Society Merit Holder. CP also would like to thank Cameron Hall for a discussion on approximations.

\section{References}

[1] Aldinger J, Klapper I and Tabor M 1995 Formulae for the calculation and estimation of writhe $J$. Knot Theory Rami. 4 343-372.

[2] Antman S 2005 Nonlinear Problems of Elasticity, 2nd ed (New York: Springer-Verlag) p 265.

[3] Asghari-Targhi M and Berger M A 2009 Writhe in the stretch-twist-fold dynamo Geophys. Astrophys. Fluid Dyn. 103 69-87.

[4] Banavar J R, Maritan A, Micheletti C and Trovato A 2002 Geometry and physics of proteins Proteins: Struct. Func. Genetics 47 315-322.

[5] Banavar J R and Maritan A 2007 Physics of proteins Ann. Rev. Biophys. Biomol. Struct. 36 261-280.

[6] Banavar J R, Cieplak M, Flammini A, Hoang T X, Kamien R D, Lezon T, Marenduzzo D, Maritan A, Seno F, Snir Y and Trovato A 2006 Geometry of proteins: hydrogen bonding, sterics and marginally compact tubes Phys. Rev. E $\mathbf{7 3}$ 031921(1-5).

[7] Bishop R L 1975 There is more than one way to frame a curve Amer. Math. Month. 82 246-251. 
[8] Brandenburg A and Subramanian K 2005 Astrophysical magnetic fields and nonlinear dynamo theory Phys. Rep. 417 1-209.

[9] Berger M A and Field G B 1984 The topological properties of magnetic helicity J. Fluid. Mech. 147 133-148.

[10] Berger M A and Prior C B 2006 The writhe of open and closed curves J. Phys. A: Math. Gen. 39 8321-8348.

[11] Călugăreanu G 1959 L'intgrale de Gauss et l'analyse des nuds tridimensionnels Czechoslovak. Math. J. 66 588-625.

[12] Călugăreanu G 1961 Sur les classes d'isotopie des noeuds tridimensionnels et leurs invariants Comm. Acad. Repub. Popul. Romine 11 829-832.

[13] Cochran W, Crick F H and Vand V 1952 The structure of synthetic polypeptides. I. The transform of atoms on a helix Acta Crystallogr. 5 581-586.

[14] Crick F H C 1953 The Fourier transform of a coiled-coil Acta Crystallogr. 6 685-689.

[15] Drenth J 1999 Principles of Protein X-ray Crystallography, 2nd ed (New York : Springer-Verlag).

[16] Coyne J 1990 Analysis of the formation and elimination of loops in twisted cable IEEE J. Ocean. Eng. 15 72-83.

[17] Fitzkee N C, Fleming P J and Rose G D 2005 The Protein Coil Library: A structural database of nonhelix, nonstrand fragments derived from the PDB Proteins: Structure, Function and Bioinfomatics 58, 852-854.

[18] Gilbert A 2002 Magnetic helicity in fast dynamos Geophys. Astrophys. Fluid Dyn. 96 135-151.

[19] Green L M, Kliem B, Török T, van Driel-Gezstelyi L. and Attrillm G.D.R. 2007 Transient coronal sigmoids and rotating erupting flux ropes Solar Phys. 246 365-391.

[20] Gonzalez O and Maddocks J H 1999 Global curvature, thickness, and the ideal shapes of knots Proc. Natl. Acad. Sci. USA, 96 4769-4773.

[21] Prudnikov A P, Brychkov Y A and Marichev O I 1988 Integrals and Series: Vol 2 (London: Gordon and Breach) pgs 37-42.

[22] Hausrath A C and Goriely A 2007 Continuous representations of proteins: Construction of coordinate models from curvature profiles J. Struct. Biol. 158 267-281.

[23] Hausrath A C and Goriely A 2009 The Fourier transforms of curves and filaments and their application to low-resolution protein crystallography J. Appl. Crystallogr. 42 268-278.

[24] Hirsch M W 1997 Differential topology (New York: Springer-Verlag).

[25] Hörmander L 1990 The Analysis of Linear Partial Differential Operators I, (Distribution theory and Fourier Analysis) 2nd ed (Berlin : Springer-Verlag).

[26] Klug H P, Alexander L E 1974 X-Ray Diffraction Procedures: For Polycrystalline and Amorphous Materials, 2nd Edition (New York: Wiley).

[27] Kournyshev A A, Lee D J, Leiken S and Wynveen A 2007 Structure and interactions of biological helices Rev. Mod. Phys. 79 943-996.

[28] Litherland R A, Durumeric O and Rawdon E 1999 Thickness of knots Topology Appl. 91 233-244.

[29] Longcope D W, Fisher G H and Arendt S 1996 The evolution and fragmentation of rising magnetic flux tubes Astrophys.J 464 999-1011.

[30] Longcope L and Klapper I 1997 Dynamics of a thin twisted flux tube Astrophys. J 488 443-453.

[31] Moffat H K 1978 Magnetic Field Generation in Electrically Conducting Fluids (London: Cambridge University Press).

[32] Moffat H K and Proctor M R E 1985 Topological constraints associated with fast dynamo action J. Fluid Mech. 154 493-507.

[33] Moreno-Insertis F and Emonet T 1996 Equilibrium of twisted horizontal magnetic flux tubes Astrophys J. 472 411-429.

[34] Nizette M and Goriely A 1999 Towards a classification of Euler-Kirchhoff filaments J. Math. Phys. 
$402830-2866$.

[35] Neukirch S and van der Heijden G H M 2002 Geometry and mechanics of uniform n-plies: from engineering ropes to biological filaments J. Elast. 69 41-72.

[36] Parker E N 1979 Cosmical Magnetic Fields: Their Origin and their Activity (Oxford : Clarendon Press).

[37] Pevstov A A 2008 What helicity can tell us about solar magnetic fields Astrophys. J. 29 49-56.

[38] Rose G D, Fleming P J, Banavar J and Maritan A A backbone-based theory of protein folding 2006 Proc. Natl. Acad. Sci. USA 103 16623-16633.

[39] Spruit H C 1981 Motion of magnetic flux tubes in the solar convection zone and chromosphere Astron. Astrophys. 98 155-160.

[40] Svergun D I and Koch M H 2002 Advances in structure analysis using small-angle scattering in solution Curr. Opin. Struct. Biol. 12(5) 654-60.

[41] Titov V S and Demoulin P 1999 Basic topology of twisted magnetic configurations in solar flares Astron. Astrophys. 351 707-720.

[42] Torok T and Kliem B 2005 Confined and Ejective Eruptions of Kink-unstable Flux Ropes Astrophys. J. Lett. 630 L97-L100.

[43] Vanishtein S I and Zeldovitch Y B 1972 Reviews of topical problems: Origin of magnetic fields in astrophysics (turbulent "dynamo" mechanisms) Sov. Phys. Usp. 15 159-172.

[44] van der Heijden G H M and Thompson J M T 2000 Helical and localised buckling in twisted rods: a unified analysis of the symmetric case Nonlinear dyn. 21 71-99.

[45] Vandiver R and Goriely A 2008 Tissue tension and axial growth of cylindrical structures in plants and elastic tissues Europhys. Lett. 84 58004(1-5). 



\section{RECENT REPORTS}

53/11 Surface growth kinematics via local curve evolution Moulton

Goriely

54/11 A multiple scales approach to evaporation induced Marangoni Hennessey convection Münch

55/11 The dynamics of bistable liquid crystal wells Luo

Majumdar

Erban

56/11 Real-Time Fluid Effects on Surfaces using the Closest Point Auer Method

Macdonald

Treib

Schneider

Westermann

$57 / 11$ Isolating intrinsic noise sources in a stochastic genetic switch

Newby

58/11 Riemann-Cartan Geometry of Nonlinear Dislocation Mechanics Yavari

Goriely

59/11 Helices through 3 or 4 points?

Goriely

Neukirch

Hausrath

60/11 Bayesian data assimilation in shape registration

Cotter

Cotter

Vialard

61/11 Asymptotic solution of a model for bilayer organic diodes and solar cells

Richardson

Please

Kirkpatrick

62/11 Neural field model of binocular rivalry waves Bressloff

Webber

63/11 Front propagation in stochastic neural fields

Bressloff

Webber

64/11 Stability estimates for a twisted rod under terminal loads: a three- Majumdar dimensional study

Prior

Goriely

65/11 Adaptive Finite Element Method Assisted by Stochastic Simulation of Chemical Systems

Cotter

Vejchodsky

Erban

66/11 On the shape of force-free field lines in the solar corona Prior

Berger

67/11 Tear film thickness variations and the role of the tear meniscus Please

Fulford

Fulford

Collins

68/11 Comment on "Frequency-dependent dispersion in porous media" Davit 
70/11 The Capillary Interaction Between Two Vertical Cylinders

71/11 Nonuniqueness in a minimal model for cell motility

Gallimore

Whiteley

Waters

King

Oliver

72/11 Symmetry of uniaxial global Landau-de Gennes minimizers in the

Henao theory of nematic liquid crystals

Majumdar

73/11 Filling of a Poisson trap by a population of random intermittent searchers

Bressloff

Newby

01/12 Mechanical growth and morphogenesis of seashells

Moulton

Goriely

Chirat

02/12 How linear features alter predator movement and the functional

McKenzie1 response

Merrill

Spiteri

Lewis

Copies of these, and any other OCCAM reports can be obtained from:

Oxford Centre for Collaborative Applied Mathematics Mathematical Institute

24 - 29 St Giles'

Oxford

OX1 3LB

England

www.maths.ox.ac.uk/occam 